\title{
Multifold Emission Enhancement in Nanoimprinted Hybrid Perovskite Metasurfaces
}

\author{
Sergey Makarov ${ }^{\S}$ Valentin Milichko ${ }^{\S}$ Elena Ushakova, ${ }^{\perp}$ Mikhail Omelyanovich, ${ }^{\S}{ }^{*}$ \\ Andrea Cerdán-Pasarán ${ }^{\$}$, Ross Haroldson, ${ }^{\dagger}$ B. Balachandran, ${ }^{\dagger *}$ Honglei Wang ${ }^{\dagger *}$, Walter Hu, ${ }^{\dagger *}$ \\ Yuri Kivshar, ${ }^{\ddagger}$, and Anvar Zakhidov ${ }^{\dagger, \S, \&}$
}

$\S$ Department of Nanophotonics and Metamaterials, ${ }^{\perp}$ Department of Optical Physics and Modern Natural Science, ITMO University, St. Petersburg 197101, Russia

* Department of Radio Science and Engineering, Aalto University, Helsinki 13000, Finland

† Nonlinear Physics Centre, Australian National University, Canberra ACT 2601, Australia

$\dagger$ Department of Physics, $\dagger^{*}$ Department of Engineering, University of Texas at Dallas, Richardson, TX 75080, USA,

\$ Universidad de Guanajuato, División de Ciencias Naturales y Exactas, Noria Alta s/n, Guanajuato, Gto. 36050, México.

${ }^{\&}$ National University of Science and Technology MISIS, Moscow, 119049, Russia

\begin{abstract}
Recent developments in the physics of high-index resonant dielectric nanostructures suggest alternative mechanisms for subwavelength light control driven by Mie resonances with strong magnetic response that can be employed for a design of novel optical metasurfaces. Here we demonstrate metasurfaces based on nanoimprinted perovskite films optimized by alloying the organic cation part of perovskites. We reveal that such metasurfaces can exhibit a significant enhancement of both linear and nonlinear photoluminescence (up to 70 times) combined with advanced stability. Our results suggest a cost-effective approach based on nanoimprint lithography and combined with simple chemical reactions for creating a new generation of functional metasurfaces which may pave a way towards highly efficient planar optoelectronic metadevices.
\end{abstract}

Keywords: metasurface, perovskites, photoluminescence, Mie resonances, nanoimprinting 
Metasurfaces emerged as two-dimensional planar structures composed of meta-atoms designed to possess special electromagnetic properties which are not found in nature [1,2]. Early realizations of such planar structures were based on noble metals and plasmonics. However, high Ohmic losses and heating of plasmonic structures motivated many researchers to look for alternative materials [3]. More recently, the study of all-dielectric resonant nanophotonic structures and metasurfaces has attracted a lot of attention being based on high-refractive-index dielectric meta-atoms which can provide many unique properties for coupling light to electric and magnetic Mie resonances in visible and near-IR ranges [4]. The corresponding all-dielectric resonant metasurfaces exhibit lowloss electromagnetic response allowing highly-efficient resonant and broadband wave-front manipulation [5,6], substantial field localization [7], as well as enhanced photoluminescence from deposited quantum dots [8] and dyes [9].

On the other hand, hybrid halide perovskites has emerged recently as a promising class of materials for advanced optoelectronic devices due to many advantages of extra-long carrier diffusion lengths, strongly suppressed carrier recombination, low trapping on defects and impurities, and high carrier mobility $[10,11]$. More importantly, hybrid organic-inorganic perovskites may provide an alternative for cost-effective fabrication and system integration, also being promising for light emitting diodes, amplifiers or lasers $[12,13]$. In contrast to many semiconductor-based active media, tuning the photoluminescence (PL) spectra of perovskites does not involve expensive techniques and requires only simple chemical reactions and proper film processing methods, such as solvent engineering or hot casting. The most common bandgap tuning procedure is to vary the halide composition with a mixture of $\mathrm{I}, \mathrm{Br}$, or $\mathrm{Cl}$, allowing to tune the positions of conduction and valence bands to desired positions within the visible and near infrared regions being important for optoelectronic applications in lasers and light emitting diodes (LEDs).

In this Letter, we suggest and develop a novel concept for creating functional metasurfaces by patterning halide perovskites with nanoimprinting technology, in order to enhance their resonant properties, as illustrated schematically in Fig 1. We employ cost-effective and large-scale nanoimprint lithography by sequential hot-mold embossing for nanopatterning perovskite spincoated films as demonstrated recently for other applications [14]. More importantly, we employ the optimized composition of perovskite thin films by alloying the organic cation part of 
perovskites, designing a composition of a triple alloy of a mixed cation in $\mathrm{Cs}_{0.5}\left(\mathrm{MA}_{0.17} \mathrm{FA}_{0.83}\right)_{0.95}$ $\mathrm{Pb}\left(\mathrm{I}_{0.83} \mathrm{Br}_{0.17}\right)_{3}$ exhibiting record photovoltaic performance, and increased stability, as was recently demonstrated in Ref. [15]. As we demonstrate below this approach of using a triple cation alloy for perovskite film, allows to avoid the degradation of nanoimprinted metasurfaces and achieve record-high PL enhancement. As a result, we suggest two-step approach to achieve highly-efficient emitting ultrathin planar structures where emission efficiency is enhanced initially by thin-film material design (at the atomic level) and then by patterning of films (at the meta-atom level). Our results pave a way towards novel cost-effective all-dielectric active planar metadevices.

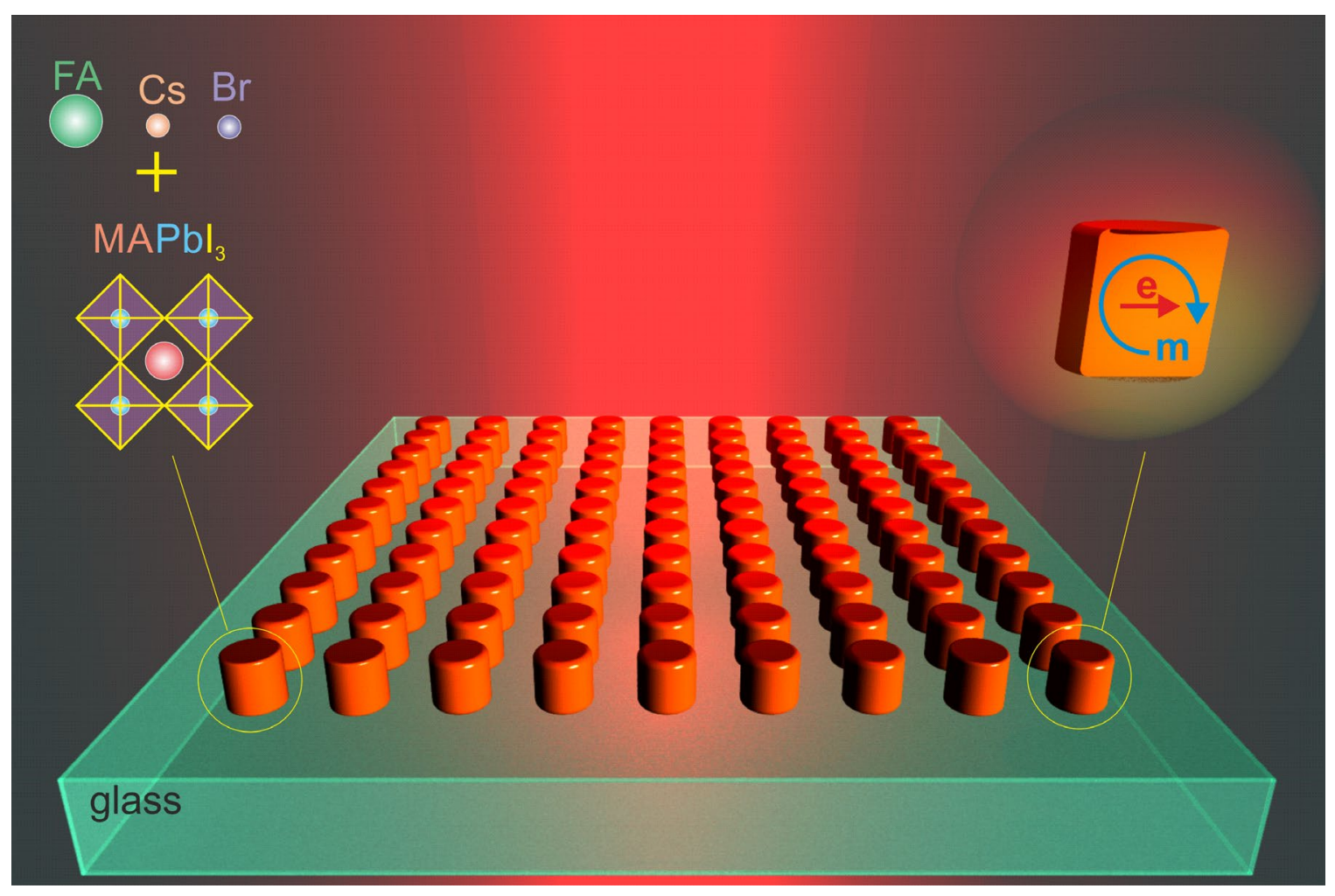

Fig. 1. General concept. Metasurfaces with enhanced functionalities achieved via structuring perovskite films, being optimized by alloying the organic cations for improved stability.

Characterization of perovskite metasurfaces. A modified solvent-engineering method [16] is employed for the deposition of perovskite thin films. This method utilizes a mixture of solvents of a perovskite for spin-coating, and then it follows with a toluene drip while spinning, which allows 
for the formation of a homogenous perovskite layer after thermal annealing. For our metasurfaces, we use fused silica for 0.5 - $\mathrm{mm}$ thick substrates. After spin-coating and toluene dripping, the sample is annealed for $10 \mathrm{~min}$ at $100^{\circ} \mathrm{C}$, which subsequently drives out the solvents and forms a perovskite film with a thickness of about $200 \mathrm{~nm}$. Samples are used later for the nanoimprinting process [14], as shown in Fig. 2. We employ two different silicon molds to compare the properties of two different types of imprinted metasurfaces. Nanopillar and nanostripe structures are first treated with an anti-adhesion monolayer of $1 \mathrm{H}, 1 \mathrm{H}, 2 \mathrm{H}, 2 \mathrm{H}$-perfluorodecyltrichlorosilane. Then, the molds are placed on the perovskite thin film on different areas in a single process to ensure the same conditions applied to all molds. The nanoimprinting is performed for $20 \mathrm{mins}$ at $100^{\circ} \mathrm{C}$ and pressure of $7 \mathrm{MPa}$. Reference samples with a spin-coated unstructured perovskite films are also prepared.

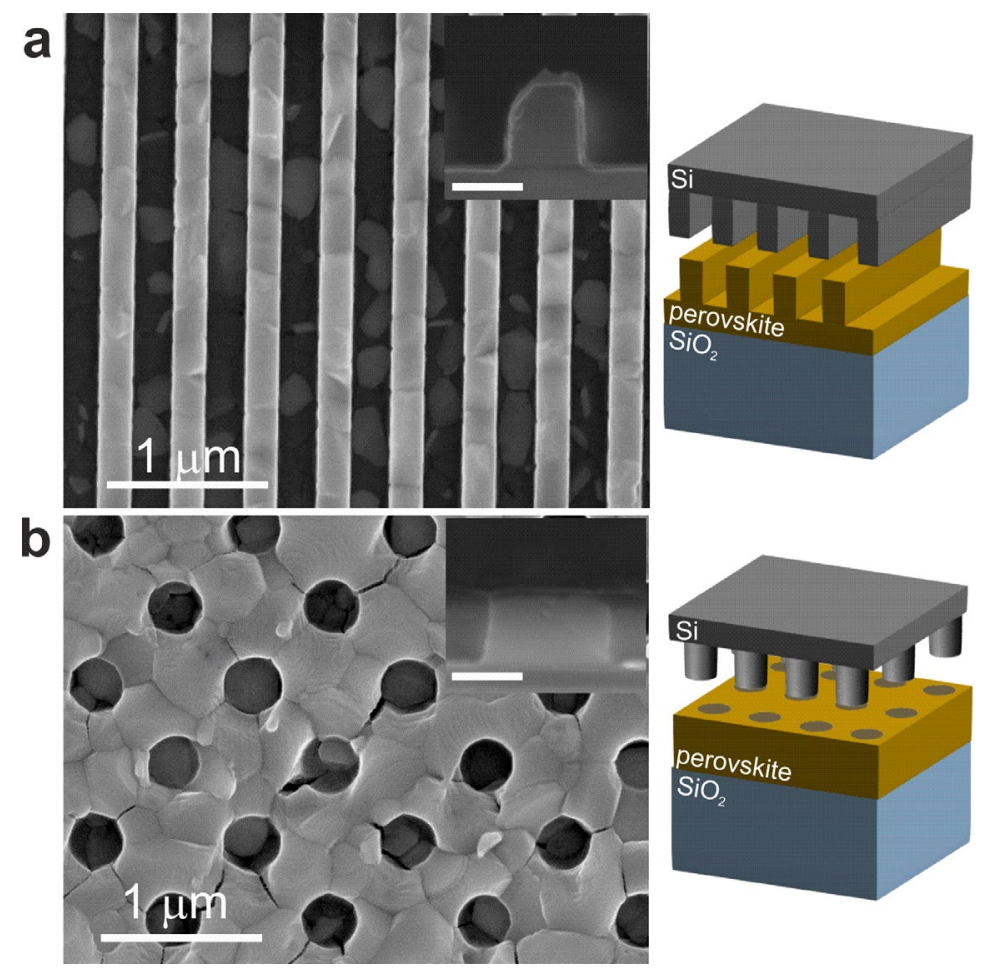

Fig. 2. Nanoimprinted perovskite metasurfaces. Scanning electron microscope images of (a) nanostripe and (b) nanohole structures. Insets show cross sections of the metasurfaces (scale bars are $300 \mathrm{~nm}$ ) and the molds used in the fabrication. 
In earlier studies of perovskite photodetectors [14], nanoimprinting of classical $\mathrm{MAPbI}_{3}$ was performed, which are known to be not very stable material in ambient atmosphere. Indeed, it is well established that $\mathrm{MAPbI}_{3}$ is notably easy degrade with heat, humidity, light, metal electrodes rather than $\mathrm{ITO}, \mathrm{Cu}$, and $\mathrm{Au}$. However, mixing $\mathrm{FA} / \mathrm{Cs}$ cations and $\mathrm{Br} / \mathrm{Cl}$ anions tends to stabilize the films. The $\mathrm{ABX}_{3}$ structure of perovskites allows for substitution of the A site cation and the $\mathrm{X}$ site halide anion while keeping the $\mathrm{B}$ site cation as $\mathrm{Pb}$. Very recently Cesium $(\mathrm{Cs})$, Formamidinium (FA), and Ethylammonium (EA) cations have been substituted in place of the Methylammonium (MA) cation to form more stable structures and tune the band gap [17-22]. Therefore, here we employ the nanoimprinting procedure to triple cation alloys of perovskites with the goal to study the effects of PL enhancement in the nanoimprinted metasurfaces of the most stable compositions with mixed Cs, MA and FA cations. Mixing these ions to form alloys in the general form of $\mathrm{Cs}_{\alpha} \mathrm{FA}_{\beta} \mathrm{MA}_{\gamma} \mathrm{Pb}\left(\mathrm{I}_{\mathrm{x}} \mathrm{Br}_{\mathrm{y}} \mathrm{Cl}_{\mathrm{z}}\right)_{3}$, where $\alpha+\beta+\gamma=1=\mathrm{x}+\mathrm{y}+\mathrm{z}$, allows us to obtain the most stable composition, and also achieve tunable band gaps. Here we are focused on one stable composition: ( $\left.\mathrm{Cs}_{0.05}, \mathrm{FA}, \mathrm{MA}\right) \mathrm{PbI}_{3-\mathrm{x}} \mathrm{Br}_{\mathrm{x}}$ with a $\mathrm{PL}$ emission in the range of $770 \mathrm{~nm}$. We compare stability of our alloy with $\mathrm{MAPbI}_{3}$ during exploiting under similar conditions for $3000 \mathrm{~h}$ and reveal strong degradation of $\mathrm{MAPbI}_{3}$, whereas $\mathrm{Cs}_{\alpha} \mathrm{FA}_{\beta} \mathrm{MA}_{\gamma} \mathrm{Pb}\left(\mathrm{I}_{\mathrm{x}} \mathrm{Br}_{\mathrm{y}} \mathrm{Cl}_{\mathrm{z}}\right)_{3}$ preserves well the PL superior properties (for details, see Fig. S1 in Supporting Information).

Morphology of the nanoimprinted films is studied by scanning electron microscopy (SEM) and presented in Figs. 2(a,b). The well-defined perovskite nanostripes [Fig. 2(a)] and nanoholes [Fig. 2(b)] structures are negative replications of the silicon molds. The nanoholes and nanostripes have a patterned diameter and period of approximately $300 \mathrm{~nm}$ and $600 \mathrm{~nm}$, respectively. The SEM cross-section reveals a depth of $315 \mathrm{~nm}$ with almost no residue layer for nanoholes, and a structure depth of $300 \mathrm{~nm}$ and residue layer depth of $85 \mathrm{~nm}$ for the nanostripes. These results demonstrate that although perovskite is an ionic solid and does not have a glass transition behavior like polymers, it can be successfully patterned by nanoimprinting methods being soft enough to deform and fill in mold cavities under applying heat and pressure. Crystallinity and surface quality improvement by nanoimprinting are also revealed by the scanning electron microscopy. This is important for carriers transport, photoluminescence lifetime and solar cell efficiency [23,24]. 
Optical properties. Reflection spectra for unpatterned and patterned perovskite films show significant modulations for nanohole and nanostripe structures [see Figs. 3(a,b)]. More specifically, we observe peaks around 1000-1100 $\mathrm{nm}$ which correspond to the excitation of optical resonances. In transmission, optical images of perovskites show different bright colors [Fig. 3(c)], suggesting reasonable uniformity of the patterning across the $1 \mathrm{~cm}^{2}$ imprint area as shown in Fig. S2 in Supporting Information.
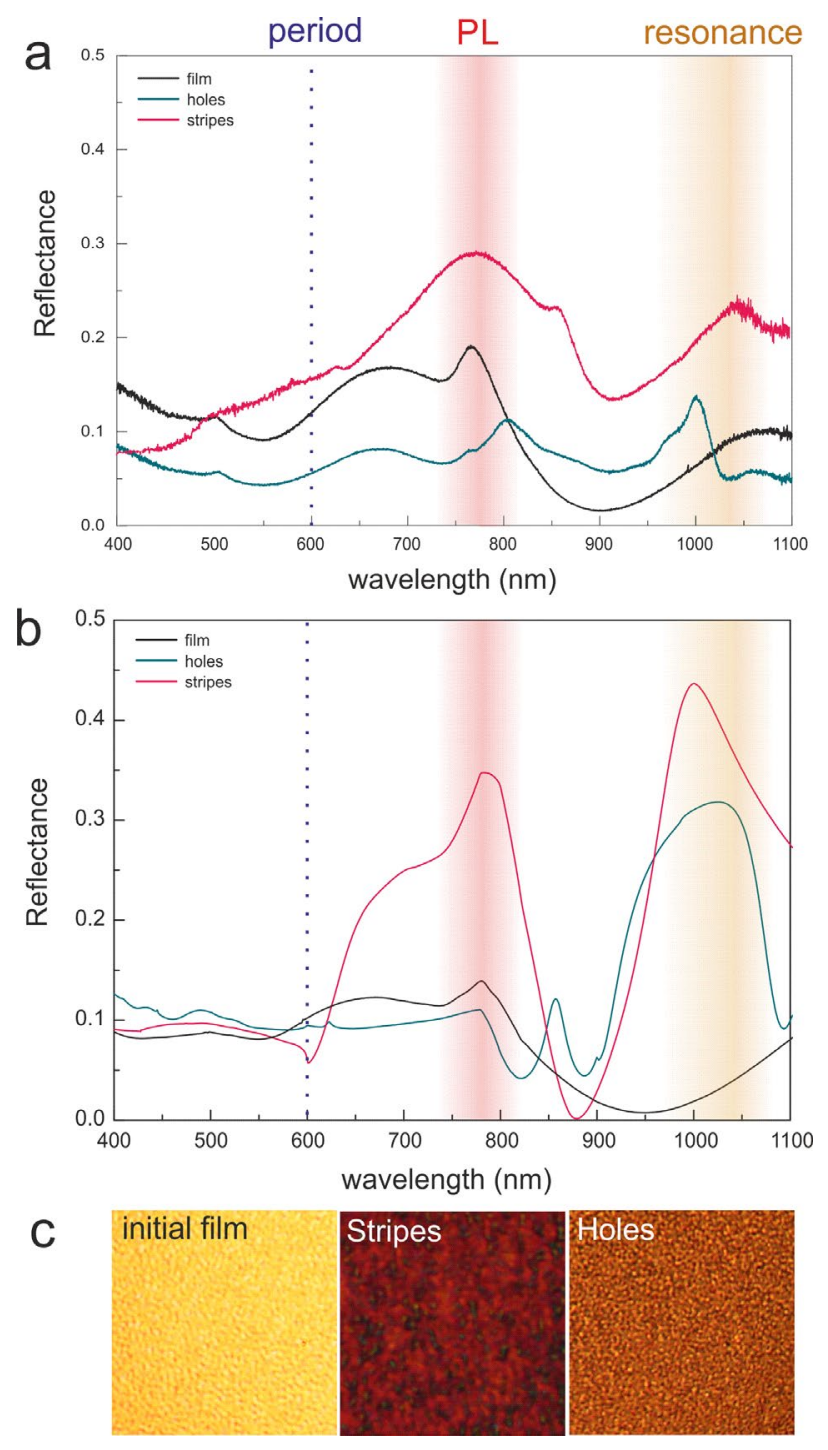

Fig. 3. Reflectance and transmittance properties of perovskite metasurfaces. (a) Experimental data and (b) numerically calculated results for the reflectance spectra of perovskite films (black curve), and metasurfaces created by nanoholes (green curve) and nanostripes (pink curve). (c) Transmittance properties: optical images of the perovskites surfaces with different patterning. 
Our numerical modeling of the reflectance of perovskite metasurfaces in CST Microwave Studio shows a qualitative agreement with the experimental spectra shown in Fig. 3(b). The details of numerical calculations are presented in Methods. Simulations of the near-fields at different frequencies reveal a resonant behavior of the nanostripe-based metasurfaces in the near IR frequency range, whereas in high-loss region $(\lambda<800 \mathrm{~nm})$, resonances are not manifested due to strong damping. In low-loss region, our patterned samples demonstrate the magnetic Mie resonances, i.e. TM modes [Fig. 4(a)]. Indeed, such a doughnut-like structure of the electric field with the four-fold field enhancement inside the materials are beneficial for applications related to nonlinear frequency conversion [25-27]. Numerical calculation of photonic band structure for the metasurfaces provides an additional information about all possible modes in such systems [see Figs. 4(c, d)], revealing a larger number of modes with high local density of states (LDOS) for nanoholes as compared with nanostripes.
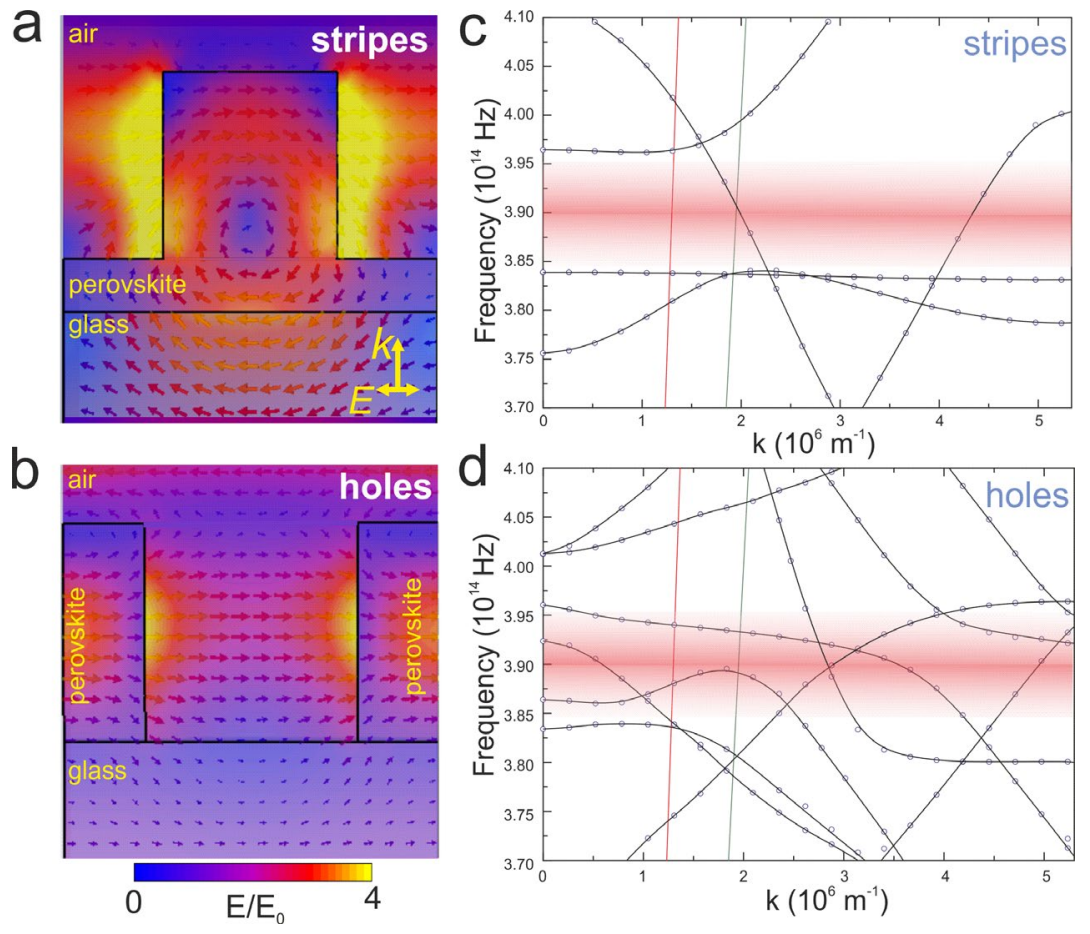

Fig. 4. Numerical results. $(a, b)$ Structure of resonant near fields of the nanoimprinted metasurfaces under the normal plane-wave incidence for experimental parameters. (c,d) Photonic bandgaps for nanostripe and nanohole metasurfaces, respectively. Almost vertical red and green lines correspond to the light cones in air and silica, respectively. Transverse shaded areas mark the PL spectral width of the perovskite. 
Photoluminescence enhancement. Beside high refractive index of the perovskite, this material demonstrates highly efficient PL at $770 \mathrm{~nm}(1.6 \mathrm{eV})$ with full-width at half maximum around 40 $\mathrm{nm}$ [Fig. 5(a)]. Therefore, it is possible to enhance PL via excitation of optical modes in the emitting material. Two specific regions can be defined: linear photoexcitation (one photon is absorbed, i.e. high optical losses at pump frequency) and nonlinear photoexcitation (multiphoton absorption, i.e. low optical losses at pump frequency).

In our experiments on linear photoexcitation, the nanoimprinted perovskites demonstrate better spontaneous emission properties with up to 2 times enhancement for nanostripes at a certain polarization and up to 8 times enhancement for nanoholes, compared with nanoimprinted thin films [Fig. 5(b)]. As shown in Fig. 5(a), these enhancement factors can be partially explained by an increase of light absorption due to nanopatterning. However, an increase of absorbed photons cannot completely describe the PL enhancement, requiring an additional analysis of the emission rate being dependent on the local density of states (LDOS).

The PL decay is acquired by using the time-correlated single-photon counting method (for details, see Methods). Measured averaged lifetime increases for imprinted nanostripes $\left(\tau_{\mathrm{g}} \approx 24.5\right.$ $\mathrm{ns})$, but decreases for imprinted nanoholes $\left(\tau_{\mathrm{h}} \approx 17.8 \mathrm{~ns}\right)$ in comparison with the perovskite thin film ( $\tau_{0} \approx 20.4 \mathrm{~ns}$ ). These times reflect both inherent changes of material (e.g., carriers' diffusion length) and an effect of resonances (e.g., Purcell factor). On one hand, nanoimprinting results in an increase of perovskite average grain size and, thus, it improves the carriers life time yielding up to 1.5 times longer PL decay [14]. On the other hand, the Purcell effect for emitting dipoles in resonator with higher LDOS leads to an acceleration of spontaneous emission. Therefore, taking into account the grain size effect, we can roughly estimate that Purcell factor averaged over the whole emission spectrum is about 2 for nanoholes, whereas it is close to 1 for nanostripes. This is in qualitative agreement with the calculated photonic band structures [Figs. 4(c,d)], where the PL spectrum covers much more states with high LDOS for nanoholes rather than for nanostripes. The Purcell factor and absorbance explain higher PL signal for nanoholes compared with nanostripes. 

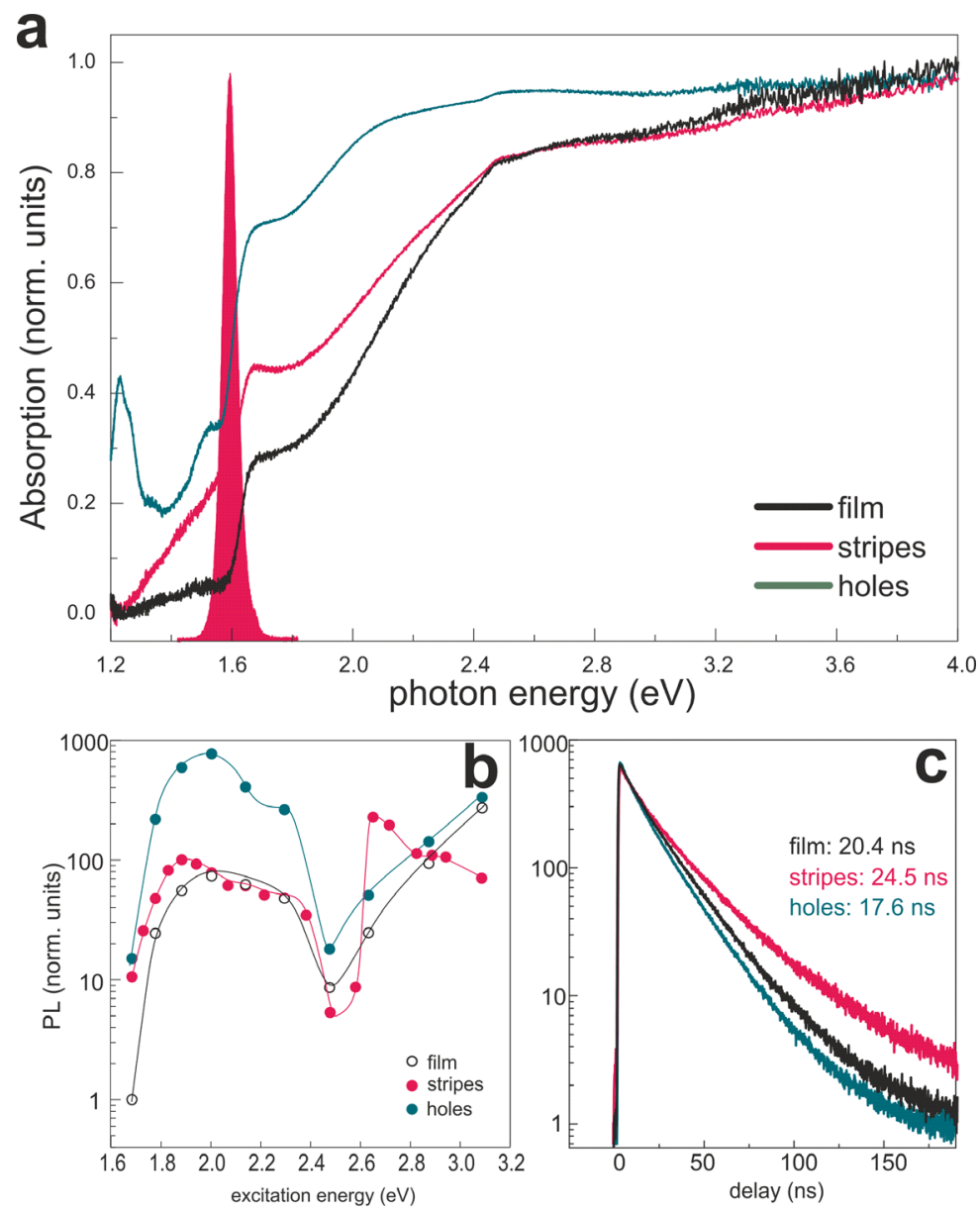

Fig. 5. One-photon photoluminescence. (a) Measured absorption spectra of a perovskite film (black), and two nanoimprinted metasurfaces (green and pink). Filled area corresponds to the PL spectrum of the perovskite. (b) PL enhancement vs energy of exciting photon. (c) Time-resolved PL measurements for three types of perovskite films

In order to study the effects of optical resonances in individual elements of nanostripes and nanoholes, we provide multiphoton PL (MPL) measurements at wavelength $\lambda=1050 \mathrm{~nm}(1.18$ $\mathrm{eV}$ ) revealing stronger dependence of a PL signal on the metasurface designs, compared with the one-photon photoexcitation. It should be stressed, that the fs-laser wavelength is significantly larger than both periods and sizes of "meta-atoms" of the imprinted nanostructures, excluding any effects of diffraction even into the silica substrates. 

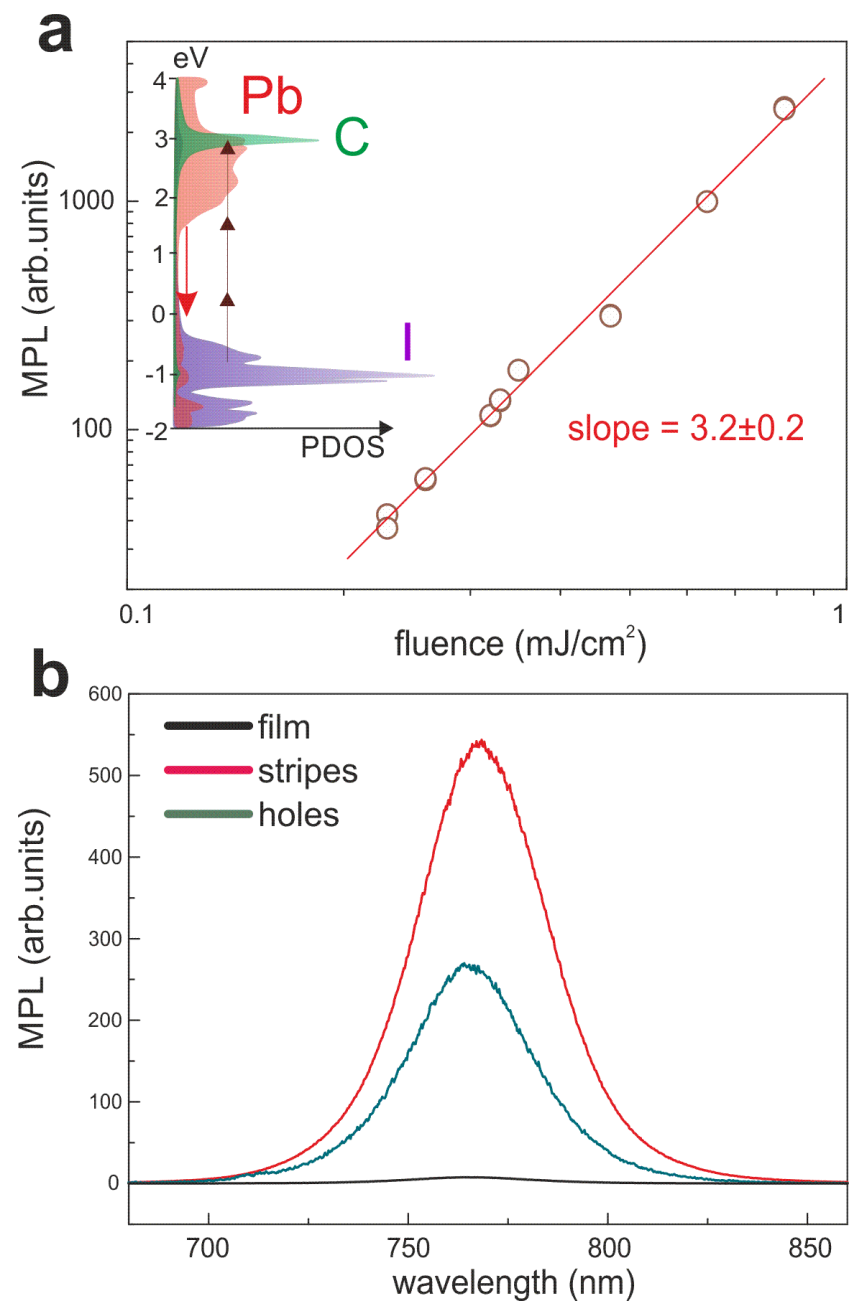

Fig. 6. Multiphoton photoluminescence. (a) MPL yield vs. average power of a femtosecond laser pulses $(\lambda=1050 \mathrm{~nm}, \tau=150 \mathrm{fs})$. Inset: schematic of absorption and emission of photons in the nonlinear regime matched with partial density of states for the perovskite basic components. (b) Comparison of MPL spectra from different types of metasurfaces.

Three-photon character of the emitted PL signal is proven by third-order slope of MPL signal dependence on the fs-laser intensity [Fig. 6(a)]. As shown in inset of Fig. 6a (adopted from Ref. [28]), the origin of effective three-photon absorption of $1.18 \mathrm{eV}$ photons can be analyzed in terms of partial density of states (PDOS), where three peaks from $\mathrm{Pb}$, I and $\mathrm{C}$ makes transitions to 3-4 $\mathrm{eV}$ much more probable rather than to $<3 \mathrm{eV}$. Moreover the recent experiments on pump-probe transient spectroscopy of perovskite, have clearly revealed a new resonance in photo-bleaching at 
$3.2 \mathrm{eV}$ in classical MAPbI3 perovskite, which has been attributed to an optical transition to a second conduction band [29]. Since our nanoimprinted perovskite film has 13\% of $\mathrm{Br}$ doping, we can expect the position of this second conduction band in our triple cation alloy slightly higher, i.e. at $\sim 3.4-3.5 \mathrm{eV}$, which coincides with energy of our 3 photon pumping by $1.18 \mathrm{eV}$ photons. This conclusion is supported by our linear measurements [Fig. 5(b)], where PL from the perovskite film is more intensive for photon energies higher than $2.8 \mathrm{eV}$. Remarkably, that spectra of one-photon and three-photon emission are shifted relative to each other, as observed previously in other studies of MPL of perovskites [30].

We believe that, the reason for stronger MPL enhancement is the dependence of three-photon PL of the studied perovskites on the local electric-field enhancement inside the material. Indeed, during multiphoton photoexcitation linear losses are very small allowing for excitation of resonances with relatively high $Q$-factors, whereas high absorption occurs only in hot-spots. In our measurements, we effectively excite the TM mode in nanostripes, as predicted by the numerical simulations [see Fig. 4(a)], when the laser polarization is orthogonal to the nanostripes. Such resonant multiphoton excitation yields 70 -fold enhancement of MPL, whereas the other polarization provides 30 times lower signal due to the absence of resonant response from the metasurface [Fig. 6(b)]. Numerical simulations of near-field around the nanoholes also reveal 3fold electric field enhancement for nanoholes at $\lambda=1050 \mathrm{~nm}$, as compared with the unpatterned film of perovskite on silica, resulting in 35-fold enhancement of MPL. It should be noted, that to describe such MPL enhancement, the Purcell effect should be taken into account.

In conclusion, we have suggested a novel approach to fabricate large-scale perovskite metasurfaces for visible and near-IR ranges with significantly enhanced PL properties. We have demonstrated that PL intensity can be enhanced up to 8 times under one-photon photoexcitation and up to 70 times under three-photon photoexcitation. We have revealed that the enhancement is achieved via the excitation of resonant Mie modes and the Purcell effect. Protection of the patterned perovskites against their degradation has been achieved by specific material preparation enabling stable metasurfaces for more than $3000 \mathrm{~h}$ in ambient media without encapsulation. Our results show that perovskites are unique materials enabling cost-effective nanoimprinting for large-scale fabrication 
of metasurfaces with high refractive index, which can be used to develop optimized planar metadevices for highly effective LEDs, lasers, and amplifiers.

\section{METHODS}

Preparation of perovskite films. The mixed-perovskite solution, $\mathrm{FA}_{\beta} \mathrm{MA}_{\gamma} \mathrm{Pb}\left(\mathrm{I}_{\mathrm{x}} \mathrm{Br}_{\mathrm{y}}\right)_{3}$, is prepared by dissolving FAI $1 \mathrm{M}, \mathrm{MABr} 0.2 \mathrm{M}, \mathrm{PbI}_{2} 1.1 \mathrm{M}$ and $\mathrm{PbBr}_{2} 0.2 \mathrm{M}$ in a 4:1 volume ratio of $\gamma$ butyrolactone (GBL) : N,N'-dimethylsulphoxide (DMSO) solvent mixture in a $\mathrm{N}_{2}$ glovebox. CsI is dissolved in DMSO to obtain $1.5 \mathrm{M}$ solution and from this, $5 \%$ volume is added to the mixedperovskite solution. The solution was heated for 12 hours at $60{ }^{\circ} \mathrm{C}$. The solution was then spincoated onto the silica substrates that was previously ultrasonic cleaned with acetone and treated by oxygen plasma. A two-step spin-coating process was performed for $25 \mathrm{~s}$ at 1000 r.p.m and then 30 $\mathrm{s}$ at 4000 r.p.m. $350 \mu \mathrm{l}$ of anhydrous toluene was dropped on the film after $12 \mathrm{~s}$ into the second spin-coating step. The sample was then annealed on a hot plate at $100{ }^{\circ} \mathrm{C}$ for 10 minutes during which solvents were evaporated and a dense and uniform film were formed with a thickness of about $200 \mathrm{~nm}$. The composition of our triple alloy perovskite film studied corresponds to a most stable structure of $\mathrm{Cs}_{0.05} \mathrm{FA}_{0.82} \mathrm{MA}_{0.13} \mathrm{~Pb}\left(\mathrm{I}_{0.87} \mathrm{Br}_{0.13}\right)_{3}$

Nanoimprinting. The $\mathrm{Si}$ nanopillars mold and nanostripes mold were first treated with $1 \mathrm{H}, 1 \mathrm{H}, 2 \mathrm{H}, 2 \mathrm{H}$-perfluorodecyltrichlorosilane (FDTS) in N-heptone solvent for 5 mins and then cleaned with acetone and blow dried with $\mathrm{N}_{2}$. The molds were then annealed at $100^{\circ} \mathrm{C}$ for 20 mins. Monolayer FDTS was formed on Si molds which served as anti-adhesive purpose in NIL process. The Si nanopillar and nanostripe molds were then placed on the perovskite thin-film coated substrate at different areas in a single process. The imprint represents a multi-step process -90 seconds at a temperature of $35^{\circ} \mathrm{C}$ and a pressure of $2 \mathrm{MPa}, 180$ seconds at a temperature of $55^{\circ} \mathrm{C}$ and a pressure of $4 \mathrm{MPa}, 180$ seconds at a temperature of $75^{\circ} \mathrm{C}$ and a pressure of $6 \mathrm{MPa}$ and then 1200 seconds at a temperature of $100^{\circ} \mathrm{C}$ and a pressure of $7 \mathrm{MPa}$. The pressure was kept at $7 \mathrm{MPa}$ while chamber was cooling down to a temperature of $35^{\circ} \mathrm{C}$. Nanoimprint process was then finished and perovskite metasurfaces were formed with negative replication of the Si molds. 
Optical characterization. All experiments were carried out at room temperature and humidity around 30\%. For optical measurements (transmission, reflection, and PL) we used a commercial confocal spectrometer Horiba LabRam HR with a cooled CCD camera (Andor DU 420A-OE 325) and $600 \mathrm{~g} / \mathrm{mm}$ diffraction grating. As sources of light we used a halogen lamp Vis-NIR HL-2000 FHSA Ocean Optics (for transmission and reflection measurements in polarized light); a supercontinuum Fianium SC400-6 with tunable wavelength of 400 to $850 \mathrm{~nm}, 60 \mathrm{MHz}$ repetition rate, pulse duration of $6 \mathrm{ps}$ and FWHM of $10 \mathrm{~nm}$ (for one-photon PL measurements in polarized light); and a femtosecond laser source (TeMa, Avesta Project) with $80 \mathrm{MHz}$ repetition rate, 1050 $\mathrm{nm}$ wavelength, pulse duration of $150 \mathrm{fs}$ and FWHM of $7 \mathrm{~nm}$ (for MPL measurements in polarized light). For transmission and PL measurements we use an objective (Mitutoyo M Plan APO 10x 0.28NA) for excitation and an objective (Mitutoyo M Plan APO NIR 50x 0.42NA) for collecting the signal. To change the excitation intensity in the MPL measurements, we use a polarizer. For reflection measurement we used an objective (Mitutoyo M Plan APO NIR 50x 0.42NA) for excitation and collecting the signal in backscattered scheme. The absorption spectra are obtained by transmission and reflection spectroscopy $(\mathrm{A}=1-\mathrm{T}-\mathrm{R})$ under the assumption that signal scattering on the crystal defects was small compared with transmission signal. All PL measurements were carried out with illumination of the samples from the bottom, i.e. through the silica substrates.

PL decay was measured by means of a laser scanning confocal microscope MicroTime 100 (PicoQuant) equipped with $10 \mathrm{x}$ objective $(\mathrm{NA}=0.25)$ and picosecond pulsed diode laser $(\lambda=405$ $\mathrm{nm}, \tau \approx 100 \mathrm{ps}$ ), which implements the method of time-correlated single photon counting. Obtained PL decay curves were fitted by biexponential function: $(t)=I_{0}+A_{1} \exp \left(-t / \tau_{1}\right)+A_{2} \exp (-t /$ $\tau_{2}$ ), where $A_{1}, A_{2}, \tau_{1}, \tau_{2}$ and $I_{0}$ are fitting amplitudes, decay times and background intensity, respectively. The average PL lifetime has been calculated with: $\langle\tau\rangle=\frac{\sum I_{i} \tau_{i}{ }^{2}}{\sum I_{i} \tau_{i}}$.

Numerical simulations. Full-wave numerical simulations of near fields were carried out by using a commercial software CST Microwave Studio employing Finite Integral Technique. To calculate dispersion laws for eigenmodes in perovskite periodical nanostructures on silica substrate, we performed a set of large scale full-vector numerical simulations with the use of finite element method solver COMSOL Multiphysics. The calculated geometries were taken from 
experimentally obtained SEM images. Dielectric permittivity of the silica substrate is 2.25 , whereas perovskite optical properties were taken from previous studies [30].

\section{ACKNOWLEDGEMENTS}

The authors thank Alexey Slobozhanyuk and Sergey Kruk for useful discussions, Danila Saranin and Artur Ishteev for help with preparing perovskite films, Yi Xin Ren for X-Ray data collection and Yi Yang and Mukti Aryal for helpful discussions on nanoimprint lithography. S.V. Makarov acknowledges the ITMO Fellowship Program. The work was partially supported by the Australian Research Council. Partial financial support from the Ministry of Education and Science of the Russian Federation (Grant № 14.Y26.31.0010 for optical measurements) and in the framework of Increase Competitiveness Program of NUST “MISiS” (No. K2-2015-014 for samples preparation) are also appreciated. We also appreciate a partial support of the Welch Foundation grant AT 1617 and CONACYT for academic opportunities and support (visiting research of A. Cerdan). 


\section{REFERENCES}

[1] N. Yu and F. Capasso. Flat optics with designer metasurfaces. Nature Materials 13, 139-150 (2014).

[2] S. Glybovski, S.A. Tretyakov, P.A. Belov, Y.S. Kivshar, and C.R. Simovski, Metasurfaces: From microwaves to visible, Physics Reports 634, 1-72 (2016)

[3] A. Boltasseva and H.A. Atwater. Low-loss plasmonic metamaterials, Science 331, 290-291 (2011).

[4] A.I. Kuznetsov, A.E. Miroshnichenko, M.L. Brongersma, Y.S. Kivshar, and B. Lukyanchuk, Optically resonant dielectric nanostructures, Science 354, aag2472 (2016).

[5] M. Decker, I. Staude, M. Falkner, J. Dominguez, D.N. Neshev, I. Brener, T. Pertsch, and Y.S. Kivshar, High-efficiency dielectric Huygens' surfaces, Advanced Optical Materials 3, 813 (2015).

[6] S. Kruk, B. Hopkins, I.I. Kravchenko, A. Miroshnichenko, D.N. Neshev, and Y.S. Kivshar, Broadband highly efficient dielectric metasurfaces for polarization control, APL Photonics 1, 030801 (2016).

[7] Y. Yang, I.I. Kravchenko, D.P. Briggs, and J. Valentine, All-dielectric metasurface analogue of electromagnetically induced transparency, Nature Communications 5, 497 (2014).

[8] I. Staude, V.V. Khardikov, N.T. Fofang, S. Liu, M. Decker, D.N. Neshev, I. Brener, and Y.S. Kivshar, Shaping photoluminescence spectra with magnetoelectric resonances in all-dielectric nanoparticles, ACS Photonics 2, 172 (2015).

[9] M. Caldarola, P. Albella, E. Cortes, M. Rahmani, T. Roschuk, G. Grinblat, R.F. Oulton, A.V. Bragas, and S.A. Maier, Non-plasmonic nanoantennas for surface enhanced spectroscopies with ultra-low heat conversion, Nature Communications 6, 7915 (2015).

[10] B.R. Sutherland and E.H. Sargent, Perovskite photonic sources, Nature Photonics 10, 295 302 (2016). 
[11] Y. Chen, H.T. Yi, X. Wu, R. Haroldson, Y.N. Gartstein, Y.I. Rodionov, K.S. Tikhonov, A. Zakhidov, X.Y. Zhu, and V. Podzorov, Extended carrier lifetimes and diffusion in hybrid perovskites revealed by Hall effect and photoconductivity measurements, Nature Communications 7, 12253 (2016).

[12] Z.K. Tan, R.S. Moghaddam, M.L. Lai, P. Docampo, R. Higler, F. Deschler, M. Price, A. Sadhanala, L.M. Pazos, D. Credgington, F. Hanusch, T. Bein, H.J. Snaith, and R.H. Friend, Bright light-emitting diodes based on organometal halide perovskite, Nature Nanotechnology 9, 687-692 (2014))

[13] H. Zhu, Y. Fu, F. Meng, X. Wu, Z. Gong, Q. Ding, M.V. Gustafsson, M.T. Trinh, S. Jin, and X.Y. Zhu, Lead halide perovskite nanowire lasers with low lasing thresholds and high quality factors, Nature Materials 14, 636-642 (2015).

[14] H. Wang, R. Haroldson, B. Balachandran, A. Zakhidov, S. Sohal, Y.X. Ren, J.Y. Chan, A. Zakhidov, and W. Hu, Nanoimprinted perovskite nanonanostripe photodetector with improved efficiency, ACS Nano (2016) in press

[15] M. Saliba, T. Matsui, K. Domanski, J.Y. Seo, A. Ummadisingu, S.M. Zakeeruddin, J.P. Correa-Baena, W.R. Tress, A. Abate, A. Hagfeldt, and M. Grätzel, Incorporation of rubidium cations into perovskite solar cells improves photovoltaic performance, Science 354, 206-209 (2016).

[16] Jeon, N. J.; Noh, J. H.; Kim, Y. C.; Yang, W. S.; Ryu, S.; Seok, S. I., Solvent engineering for high-performance inorganic-organic hybrid perovskite solar cells, Nature Materials 13, 897-903 (2014).

[17] Z. Yang, C. Chueh, P. Liang, M. Crump, F. Lin, Z. Zhu, and A. Jen, Effects offormamidinium and bromide ion substitution in methylammonium lead triiodide toward high-performance perovskite solar cells, Nano Energy 22, 328-337 (2016).

[18] M. Mittal, Atanu J., S. Sarkar, P. Mahadevan, and S. Sapra, Size of the organic Cation tunes the band gap of colloidal organolead Bromide perovskite nanocrystals, J. Phys. Chem. Lett. 7, 3270-3277 (2016) 
[19] Y. Deng, Q. Dong, C. Bi, Y. Yuan, and J. Huang, Air-Stable, Efficient Mixed-Cation Perovskite Solar Cells with Cu Electrode by Scalable Fabrication of Active layer, Adv. Energy Mater. 6 (2016), DOI: 10.1002/aenm.201600372

[20] M. Saliba, T. Matsui, J. Seo, K. Domanski, J. Correa-Baena, M. Nazeeruddin, S. Zakeeruddin, W. Tress, A. Abate, A. Hagfeldt, and M. Grätzel, Cesium-containing triple cation perovskite solar cells: improved stability, reproducibility and high efficiency, Energy Environ. Sci. 9, 1989-1997 (2016).

[21] D. Mcmeekin, G. Sadoughi, W. Rehman, G. Eperon, M. Saliba, M. Hörantner, A. Haghighirad, N. Sakai, L. Korte, B. Rech, M. Johnston, L. Herz, and H. Snaith, A mixed-cation lead mixed-halide perovskite absorber for tandem solar cells, Science 351, 151-155 (2016)

[22] R. Beal, D. Sotcavage, T. Leijtens, A. Bowring, R. Belisle, W. Nguyen, G. Burkhard, E. Hoke, and M. McGehee, Cesium Lead Halide Perovskites with Improved Stability for Tandem Solar Cells, J. Phys. Chem. Lett. 7, 746-751 (2016)

[23] D.W. de Quilettes, S.M. Vorpahl, S.D. Stranks, H. Nagaoka, G.E. Eperon, M.E. Ziffer, H.J. Snaith, and D.S. Ginger, Impact of microstructure on local carrier lifetime in perovskite solar cells, Science 348, 683-686 (2015).

[24] Xiao, J.; Yang, Y.; Xu, X.; Shi, J.; Zhu, L.; Lv, S.; Wu, H.; Luo, Y.; Li, D.; and Meng, Q., Pressure-assisted CH3NH3PbI3 morphology reconstruction to improve the high performance of perovskite solar cells. Journal of Materials Chemistry A 3, 5289-5293 (2015).

[25] M.R. Shcherbakov, D.N. Neshev, B. Hopkins, A.S. Shorokhov, I. Staude, E.V. MelikGaykazyan, M. Decker, A.A. Ezhov, A.E. Miroshnichenko, I. Brener, and Y.S. Kivshar, Enhanced third-harmonic generation in silicon nanoparticles driven by magnetic resonances, Nano Letters 14, 6488-6492 (2014).

[26] P.A. Dmitriev, D.G. Baranov, V.A. Milichko, S.V. Makarov, I.S. Mukhin, A.K. Samusev, A.E. Krasnok, P.A. Belov, and Y.S. Kivshar, Resonant Raman scattering from silicon nanoparticles enhanced by magnetic response, Nanoscale 8, 9721-9726 (2016). 
[27] R. Camacho, M. Rahmani, S. Kruk, L. Wang, L. Xu, D. Smirnova, A. Solntsev, A. Miroshnichenko, H.H. Tan, F. Karouta, S. Naureen, K. Vora, L. Carletti, C. De Angelis, C. Jagadish, Y.S. Kivshar, and D.N. Neshev, Nonlinear generation of vector beams from AlGaAs nanoantennas, Nano Letters (2016) DOI: 10.1021/acs.nanolett.6b03525

[28] M. Kato, T. Fujiseki, T. Miyadera, T. Sugita, S. Fujimoto, M. Tamakoshi, M. Chikamatsu, and H. Fujiwara, Universal rules for the visible-light absorption in hybrid perovskite materials, arXiv: 1605.05124 (2016).

[29] B. Anand,1 S. Sampat, E. O. Danilov, W. Peng, S. M. Rupich, Y.J. Chabal, Y. N. Gartstein, and A. V. Malko, Broadband transient absorption study of photoexcitations in lead halide perovskites: Towards a multiband picture, Phys. Rev. B 93, 161205(R) (2016)

[30] Y. Wang, X. Li, X. Zhao, L. Xiao, H. Zeng, and H. Sun, Nonlinear absorption and lowthreshold multiphoton pumped stimulated emission from all-inorganic perovskite nanocrystals, Nano Letters 16, 448-453 (2015).

[31] J.M. Ball, S.D. Stranks, M.T. Hörantner, S. Hüttner, W. Zhang, E.J. Crossland, I. Ramirez, M. Riede, M.B. Johnston, R.H. Friend, and H.J. Snaith, Optical properties and limiting photocurrent of thin-film perovskite solar cells, Energy \& Environmental Science, 8, 602-609 (2015).

\section{TOC image}
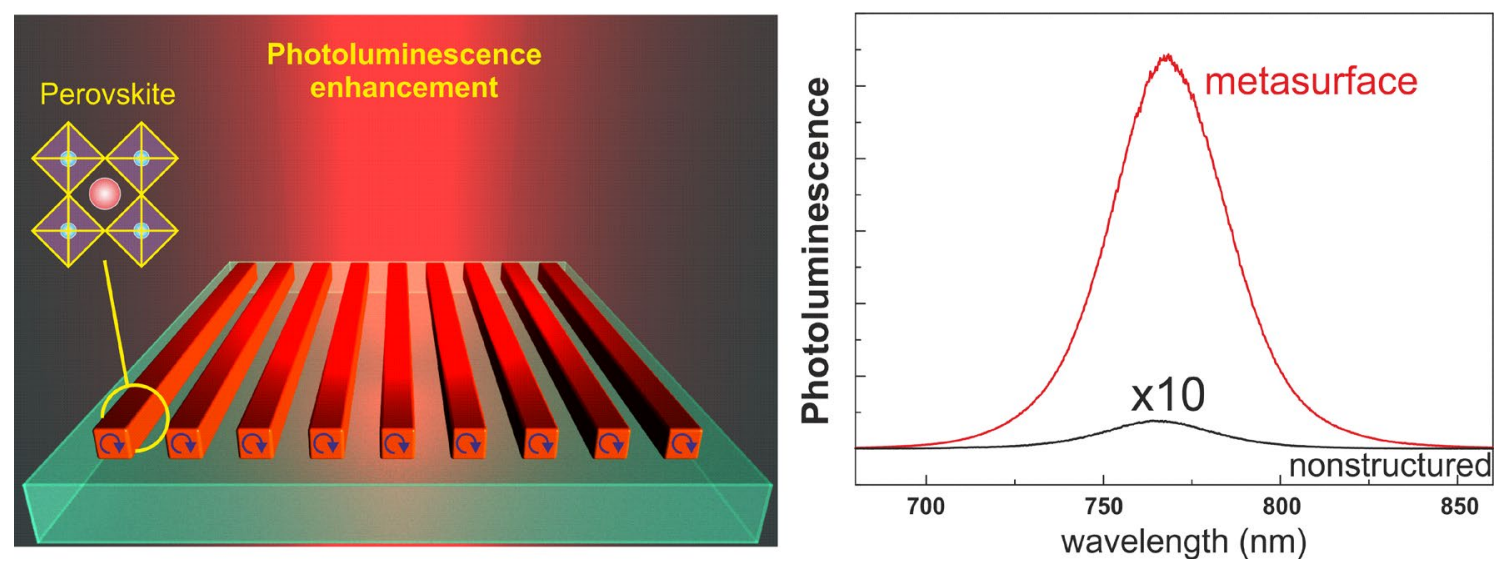